\title{
EDITORIAL
}

Giuseppe Piccardo • Francesco D'Annibale • Daniele Zulli

\section{On the contribution of Angelo Luongo to Mechanics: in honor of his 60th birthday}

Published online: 5 November 2014

(c) Springer-Verlag Berlin Heidelberg 2014

\begin{abstract}
This paper intends to summarize the scientific production of Angelo Luongo on the occasion of his Sixtieth Birthday, focusing on his main contributions in the field of Mechanics. The task will not be easy because of the breadth of his scientific production, only apparently attributable to a restricted number of keywords. In fact, even when the work seems purely algorithmic, speculation on the physical and mechanical aspects of the problem is always present, providing new interpretations and innovative openings to a careful reader. Similarly, also the works, which apparently seem to be high-level applications, always reserve methodological aspects that are not negligible. The editorial choice to divide his papers through a small number of keywords is certainly simplistic, but offers the possibility to better highlight all the connections among his variegated production. The most original contributions of Angelo Luongo in the context of perturbation methods, linear and nonlinear dynamics and control, elastic buckling and structural analysis, bifurcation and stability of non-conservative systems, are discussed in detail. Finally, the Angelo Luongo's central role in the creation and development of activities of the international research center M\&MoCS is pointed out.
\end{abstract}

Keywords Perturbation methods · Nonlinear dynamics · Bifurcation and stability · Structural modeling · Dynamics and control · Elastic buckling

\section{Introduction}

The scientific activity of Professor Angelo Luongo, in his first sixty years of life, has been characterized by some very peculiar features: independence of thought, deep knowledge of specific mathematical tools, originality of his results, deep physical understanding of considered problems, great variety of treated subjects. He also deeply influenced even elder co-authors imposing his creative originality and far reaching vision of nonlinear phenomena and their mathematical modeling. However, the most important contribution to mechanical science

Communicated by Francesco dell'Isola.

G. Piccardo $(\varangle)$

DICCA, Polytechnic School, University of Genoa, Via Montallegro 1, 16145, Genoa, Italy

E-mail: giuseppe.piccardo@unige.it

F. D'Annibale

M\&MoCS, International Research Center on Mathematics and Mechanics of Complex Systems,

University of L'Aquila, L'Aquila, Italy

F. D’Annibale · D. Zulli

DICEAA, University of L'Aquila, Via Giovanni Gronchi, 67100 L'Aquila, Italy

E-mail: francesco.dannibale@univaq.it

D. Zulli

E-mail: daniele.zulli@univaq.it 
is the large number of scientists who have been formed, up to the present days, under his scientific guidance: this will be his true legacy. A brief presentation of his intense academic activity and scientific vision is set out in the 'Appendix.'

This paper aims to highlight contributions of Angelo Luongo to Mechanics classifying his scientific work, a task by no means easy. His entire production could be summarily described by a few keywords: Perturbation Methods, Nonlinear Dynamics, Bifurcation and Stability, and Structural Modeling. Such a sorting, however, is not entirely satisfactory since most of his papers do not relate to just one of these categories.

Also, a classification based on the theoretical/applicative contributions is not entirely suited. Indeed, even when the algorithmic aspects constitute the main focus of the work, the physical speculation is never absent, and vice versa. As a matter of fact, many of the most brilliant insights he had about implementation of new perturbation methods were suggested by the need to respond to a mechanical problem. However, the opposite is also true as will be discussed later.

Even the antithetic distinction between static/dynamic approaches does not apply to his papers. In an early stage of his career, he worked on elastic stability, but soon he addressed to Nonlinear Dynamics and, successively to the (Dynamic) Stability of Non-Conservative System. In this evolution, he merged the know-how of the 'static perturbation analysis,' well known in the 80's to civil engineers, and the knowledge of perturbation methods, well known to mechanical engineers in the same period, to build up a complex of (perturbation) methods for the analysis of dynamic stability, which nowadays are alternative to the more popular Center Manifold and Normal Form approach. In other words, he has built a bridge between two communities, the 'static' and the 'dynamic' ones, which until then had had very few exchanges, by taking advantage of the relevant studies. Some emblematic papers deal with both the aspects, dynamics and buckling, to underline their common roots in his mind.

Also, the division linear/nonlinear does not work in classifying his work. He often affirmed that the perturbation methods (the fil rouge of his production) make no distinctions among them since, in that view, nonlinearities play the same role than imperfections (or modifications) play in linear systems. Thus, he exploited a formal analogy between the perturbative equations for (linear) eigenvalue sensitivity and the (nonlinear) bifurcation equations to implement new algorithms for these latter.

Due to all these considerations, the authors of this review article tried several ways to describe the papers by Angelo Luongo, most of them unsatisfactory. Finally, they decided to come back to the first idea of keywords, certainly simplistic but able to better highlight all the connections among his variegated production. Thus, the review article dedicated to Professor Angelo Luongo is organized as follows. In Sect. 2 papers in which the formulation of perturbation algorithms prevails are summarized. In Sect. 3 treatments relevant to linear and nonlinear dynamics, as well as control, are illustrated. In Sect. 4 contributions mainly addressed to the field of elastic buckling, structural modeling, and localization, both in statics and dynamics, are commented. In Sect. 5 the largest set of papers dealing with bifurcation and stability of non-conservative systems is structured in methodological and applicative treatments, depending on their prevailing context. A concluding section on advances and perspectives of the researches inspired or coordinated by Angelo Luongo at the International Centre M\&MoCS ends the article.

Closing these introductory notes, we want to recall an anecdote that Angelo Luongo told to his student several times, during seminars and lectures. At the begin of the 90', he was attracted by defective (nil-potent) matrices, since standard perturbation methods seemed to fail when applied to them. The research was exclusively 'curiosity driven,' without any application in mind. One day, while he was working on this subject, with his desk overwhelmed by handwritten calculations, a colleague of him (a well-known, world-renowned scientist) asked: "Why are you interested in studying singular cases that you will never meet in your life?". He answered: "You cannot know now what you will need tomorrow." Well, from then on, Angelo Luongo exploited the knowledge on defective matrices to successfully analyze several types of defective bifurcations (the most likely to occur, in the field of non-conservative systems, when the codimension is larger than 1). Angelo Luongo behaves consistently with the well-known idea of bifurcation theory, according to which singularities of a system are important since they organize the dynamics of their neighborhood. Indeed, he represents a singularity in our little scientific circle and, as his beloved singular systems, he has strongly influenced not only his pupils but all the people who have been close to him in these years.

\section{Perturbation methods}

In a group of papers [1-8] some general techniques, relative to perturbation methods, have been considered and revisited under the boost arising from various applications dealt with in the other papers. 
1. A perturbation technique based on series expansions of non-integer powers of the small parameter is developed for systematically computing the directional eigenderivatives of defective matrices [1], highlighting the marked sensitivity of eigensolutions and explaining the mechanism for generating a complete system of eigenvectors from an incomplete one. These pathological systems are discussed in [2], showing that mechanical defective systems can really exist if non-conservative forces are taken into account; moreover, their main characteristics mainly depend on the strength of non-conservative forces, and they are represented not by isolated, special points but rather by curves or surfaces in the parameter space (i.e., oneparameter or multi-parameter systems). Defective systems, however, represent exceptional cases. More often systems having close eigenvalues are encountered (mistuned systems) instead of perfectly coincident (tuned systems), originating cases of nearly defective systems if the associated eigenvectors make groups of nearly parallel vectors. Standard methods for eigenvalue sensitivity analysis fail for such systems, as discussed in [3]. To overcome the problem, one has first to find an ideal defective system close to the nearly defective and then to start perturbation from the former. An algorithm for building up a suitable tuned problem when the eigensolutions are nearly coincident in twos is developed in [3]. There, an interesting geometric interpretation of singular perturbations is also given: they occur when the modification is tangent to the locus of the defective systems in the space of parameters.

2. Perturbation approaches, such as the Lindstedt-Poincare and Multiple Scale Methods (MSM), are generalized to deal with nonlinear autonomous discrete-time systems, paying particular attention to almostperiodic and transient solutions [4]. The proposed methods make use of shift operator transformation laws (that can be considered as the counterpart for discrete systems of the chain rules valid for continuous systems) which allow the derivation of perturbation equations in a systematic way. The discrete asymptotic solutions tend to the asymptotic continuous ones when the time step tends to zero.

3. Significant advances in the analysis of multi-resonant systems by the Multiple Scale Analysis are proposed, which result in various benefits in all subjects addressed, including applications. The study of codimension$M$ bifurcations for finite-dimensional autonomous systems is summarized in [5] where eigenvalue sensitivity analysis is presented suggesting strategies for solving nonlinear bifurcation problems. Some algorithmic aspects of MSM for both non-defective and defective bifurcations are pointed out leading to fundamental rules concerning the reconstitution procedure. A geometrical approach to the problem of evaluating the classes of motion in general multi-resonant systems is illustrated in [6]. It consists in the following two steps: the set of the classes of motion admitted by each individual resonance (family) is first built up; then, the interactions among families are studied recursively in pairs by applying simple rules generated by a unique theorem. The method furnishes class diagrams which synthesize the coupling existing among the amplitudes as well as their importance in motion description. Moreover, three types of perturbation have been identified in the structure of variational equations governing the stability of steady solutions: in-class perturbations, i.e. perturbation of amplitudes participating in the motion; non-resonant and resonant outof-class perturbations, i.e. perturbations of the amplitudes not directly participating in the motion or not involved in resonance with the active amplitudes, respectively.

4. Relevant studies on the general structure of the amplitude modulation equations (AME), furnished by the Multiple Scale Analysis or equivalent perturbation procedures, are carried out. An extensive analysis of alternative forms of the AME, governing the asymptotic dynamics of multi-resonant systems, is presented [7]. AMEs are derived under general simultaneous internal and external resonance conditions, using different representations for complex amplitudes; their effectiveness in analyzing periodic motions is proven. The standard form for the Reduced AMEs (RAMEs) is deduced, although under restrictive conditions, by using rotating bases and a mixed polar-Cartesian representation. Moreover, rules to construct the qualitative form of the AMEs to any desired order are discussed in [8]. Two families of terms are identified: improper resonant terms (not associated at any resonance conditions) and proper resonant terms, which can be subdivided into primary (of lower order) and secondary (of higher order) terms. Theorems are proved to show that both improper and secondary resonant terms have no qualitative but only quantitative effects on classes of motion. An algebraic algorithm is illustrated to determine the classes of motion, and the concept of degree of constraint of a given resonance condition is introduced.

\section{Dynamics and control}

This section is devoted to frame the papers dealing with problems in linear, nonlinear dynamics and control, for both discrete and continuous systems. 


\subsection{Linear dynamics}

Although his research focus is mainly devoted to nonlinear dynamics and its implications, contributions to linear dynamics are not negligible. In the papers $[9,10]$, a theme that will resurface often in the scientific production of Angelo Luongo appears for the first time: the dynamical behavior of cables. In particular, in [9] a finite-difference algorithm is introduced to analyze the natural frequencies and modal shapes of a linear elastic cable with moving supports, highlighting the crucial role of the cable extensibility in the correct caption of the the crossover phenomenon, which substantially appears as a matter of kinematic compatibility. It is remarkable that the paper [10] deals with a critical review of a previous contribution in the field: some important comments concerning the physical insight into free vibration of parabolic cables and their frequency spectrum are provided highlighting his growing competence in this topic that will lead to some key papers on cable nonlinear dynamics.

Some basic results regarding periodic structures are contained in [11-13]. In particular, general bi-coupled periodic systems are dealt with by means of transfer matrices of single units [11]. A qualitative analysis carried out on the invariants' plane is proposed with an exhaustive description of the free wave propagation patterns. It enables both to identify the stop, pass, and complex domains and to analytically derive the boundaries of such regions, considering symmetric and unsymmetrical structures made up of beams resting on elastic supports, with both distributed and lumped masses. Based on these results, aiming at reducing the transmitted vibrations, a design of optimal piecewise periodic structures is proposed in [12]. The periodic cell properties and arrangement are tailored to localize the response around the excitation source within any assigned frequency range. The amount of vibration suppression along the periodic structure is controlled and can be described through iso-attenuation curves representing the contour plot of the real part of the propagation constants. Despite these advances, numerical difficulties to determine natural frequencies when solving the complex determinant resulting from the wave vector approach may arise. The causes of such drawbacks are investigated in [13], showing that numerical difficulties are due to the ill-posed mathematical problem in terms of complex waves coming on the structure. According to this classic method, natural frequencies are found as real solutions of complex characteristic equations, i.e. as a solution of a system of two equations in just one unknown. Aiming to overcome such problems while retaining the advantages of the wave transfer matrix framework, a modified version of the classic wave vector computational scheme is proposed dealing with real quantities only, thus avoiding the described pathology.

\subsection{Nonlinear dynamics}

The nonlinear dynamics of elastic or suspended cables, shear indeformable beams, tethered satellite systems, orbiting strings, chains of sliding beams and pendulums has been dealt with in the papers [14-27]. Very often making use of perturbation methods on reduced systems of ordinary differential equations relevant to the different structures involved in the papers, original contributions can be summarized in the following points.

1. Concerning sagged suspended cables, a two degree-of-freedom (DOF) nonlinear elastic model is proposed to analyze the effects of nonlinearities on the free motion in absence of internal resonance [14] using the multiple scale perturbation method. This model differs from those proposed hitherto for the presence of quadratic nonlinearities together with cubic ones. The conditions under which extensional in-plane and pendulum out-of-plane mono-frequent oscillations exist are examined as well as those for which effects arise due to the nonlinear coupling. A milestone in the study of planar nonlinear free vibrations of suspended cables is represented by [15], where nonlinear equations of motion of a heavy elastic cable about a deformed static equilibrium configuration are developed through a Lagrangian description of the continuum problem in the context of dynamics of prestressed solids. After introducing consistent simplifying assumptions (including the static condensation of the longitudinal displacement component), the partial differential equations of planar motion are reduced to one ordinary equation via a Galerkin procedure, pursuing an approximate solution through the Lindstedt-Poincare method. Moreover, the possibility of introducing a dimensionless form of the equations suitable to describe the nonlinear oscillations of a cable in terms of a unique parameter characterizing its mechanical and geometrical properties, as it occurs in linear dynamics, is analyzed. The discussion in [16] on large amplitude vibrations of cables states the peculiar aspects of the nonlinear dynamic behavior of sagged cables compared to taut strings, because of the curvature of the initial configuration which leads to quadratic nonlinearities. 
2. Regarding the behavior of compact and non-compact inextensional beams, [17] proposes a unified approach to study planar forced oscillations of shear indeformable beams for the two different cases of movable and fixed support at one end, starting from an exact nonlinear model subsequently restricted to third-order nonlinearities and reduced to one nonlinear differential equation. An internal constraint is introduced for systematically eliminating the longitudinal displacement. With the background of this case concerning a weak nonlinear behavior, strong nonlinear interactions among transversal and torsional vibrations of non-compact beams are studied in [18]. The problem is accurately described by three equations in the two transversal displacements and torsion; they include the quadratic and cubic terms of elastic interaction among flexural and torsional components but do not contain warping since its consistent introduction is far from being straightforward in this kind of nonlinear beam models. A perturbation solution highlights relevant parameters governing the phenomenon: when a resonance condition involving quadratic nonlinearities is approached, even though one motion component is assigned a zero initial value, it is forced strongly in the motion and, in turn, modifies the laws of the remaining two. The nonlinear effects of warping and torsional elongation are considered in [19], examining nonlinear three-dimensional oscillations of beams in internal resonance conditions, with a cross-section having a symmetry axis. In some particular situations, nonlinear warping and torsional elongation effects can produce deep modifications in the dynamic behavior of the beam from both a quantitative and a qualitative viewpoint.

3. Research field on tethered satellite systems is inspired by the investigation of their free dynamics during the station-keeping phase. Since out-of-plane oscillations are governed by equations uncoupled from in-plane motion, [20] analyzes the in-plane motion where longitudinal and transversal displacements are coupled by means of gyroscopic forces, leading to a nonstandard eigenvalue problem. New contributions arise from the proposal of a more sophisticated model and the use of perturbation techniques in computing problem eigensolutions. Reference [21] presents the equations of motion developed in a Taylor series up to the third order, so as to obtain a set of equations that can be suitably solved by perturbation techniques. In this way, it is possible to consider the modification of the frequency and of the oscillation shape with amplitude, rarely taken into account in similar studies. This fact appears very important since a number of modes are usually involved due to conditions of internal resonance of tethered systems. In particular, the modification of frequency with amplitude, which would be negligible in a weakly nonlinear system, is, however, appreciable since it is always associated with a modification in the shape of oscillation, due to internal resonance conditions characterizing this orbiting systems. In [22], attention is focused on non-stationary motions in conditions of simultaneous internal resonances. A two-mode model can give an approximate description of oscillations in primary 1:2 resonance; richer models are needed to study more complex motions, such as simultaneous internal resonance 2:4:1 and 1:2:1. With the aim of studying stability and non-stationary motions arising from perturbed two-mode non-planar oscillations, a four-mode model is developed in [23] since at least two modes are necessary to describe the perturbation of basic oscillations. The stability of steady and periodic solutions is analyzed by introducing a Cartesian representation of complex amplitudes; the stability of periodic motions is governed by a system of variational equations with periodic coefficients.

4. An approximate explicit response probability density function of a simply supported beam under external and axial impulsive random Poisson excitation is deduced [24]. The impulsive loading is a process having Dirac delta occurrences with random intensity distributed in time according to Poisson's law. The proposed approach considers parametric excitation that is multiplicative in displacement since the integral term appearing in the Kolmogorov-Feller equation offers a better opportunity to find approximate solutions.

5. The analytical determination of nonlinear transfer function and frequency-amplitude relationship for chain of nonlinear beams resting on sliding supports has been determined in [25], in order to detect their free and harmonically forced dynamical behavior, after exploitation of some periodicity conditions. To determine the dynamic response of the system, the transfer-matrix approach is merged with the harmonic balance method. In analogy to the linear case, the approach leads to an algebraic set of equations whose dimensions are equal to the number of coupling degrees of freedom.

6. The detection of analytical expressions for nonlinear free-vibration frequencies and mode shapes in case of a breathing-cracked beam, modeled as a two DOF system, is dealt with in [26] proposing a modification of the classical Lindstedt-Poincaré method, able to tackle non-smooth piecewise linear systems. Differently for the classic approach, valid for smooth systems, the complementary solution of the passive coordinate equations must be taken into account in order to satisfy continuity and periodicity of motion; accordingly, both passive and active frequencies contribute to the motion although they are incommensurable. 
7. The characterization of the dynamics of a pendulum with periodically varying length, in terms of steady dynamics and limit cycles, as well as non-regular and chaotic motions, is studied in [27]. Unfairly little studied in the literature, this example exhibits a variety of types of behavior and can be naturally treated as a model of swing. Comparisons with the pendulum having oscillating support and fixed length show the distinctive dynamical features of pendulum with periodically varying length such as high-speed regular rotations and absence of second instability domain.

\subsection{Control}

In the series of papers [28-35] interesting results about control of oscillations for many different systems can be found: tethered satellite systems, aeroelastic oscillators and tuned mass dampers, double pendulum, and nonlinear energy sinks.

1. Tethered orbiting systems are characterized by weak nonlinearities; notwithstanding their nonlinear dynamics offer interesting points to be investigated due to internal resonance conditions, which may produce unstable oscillations. In fact, the system exhibits quadratic nonlinearities, and the frequencies of flexible modes are almost in integer ratio. Reference [28] proposes the use of the longitudinal force as a control parameter in order to reduce the primary and secondary instability regions occurring in the presence of internal resonances. Since the displacement component in the orbit plane is always present in the motion due to nonlinear coupling, the control force can be considered as a function of in-plane displacements only, also when a prevailing out-of-plane oscillation occurs. Reference [29] points out that a linear velocity feedback does not produce damping but modifies the nonlinear terms only with satisfactory effects on the stability regions; on the other hand, a quadratic velocity feedback produces cubic damping terms able to suppress oscillations and to eliminate the primary mechanism of energy transfer from in-plane and out-of-plane motion.

2. About aeroelastic oscillators and tuned mass dampers, the control of a single DOF system under aerodynamic forces inducing galloping is dealt with [30] considering the flow-structure interaction as the only source of nonlinearity. Critical conditions for occurrence of simple Hopf bifurcation as well as non-resonant and 1:1 resonant double Hopf bifurcations are detected. It is shown that the effectiveness of tuned mass dampers persists even in the post-critical range. For the same model, deep unfolding of the post-critical dynamics for the most cumbersome case, namely the 1:1 resonant double Hopf bifurcation, is provided in [31]. The entire post-critical scenario in the bifurcation parameter space is analyzed highlighting the limits of validity of the equivalent single DOF concept. It is shown that, if the control parameters maximize the critical wind velocity, then the limit cycle amplitude also reaches a minimum; therefore, the optimal tuned mass damper retains its peculiarities in the nonlinear range too. Moreover, since all the control parameters are determined by the required optimal conditions, no other conditions are available to improve the post-critical performance. Thus, a suitable nonlinearity could be introduced in the system to reduce wind effects after the critical condition. A preliminary study of the effectiveness of a nonlinear cubic damping in tuned mass dampers on the bifurcation scenario of a simple aeroelastic oscillator is explored in [32], pointing out the existence of a better nonlinear tuned mass damper in a region sufficiently far from the optimal linear case.

3. In [33], the challenging problem of the stabilization of the upright statically unstable position of a double pendulum is pursued through a parametric excitation, focusing the interest in the obtaining of both lower and upper bounds of the stability regions in the plane of parameters. Non-straightforward perturbation algorithms have been implemented with a proper ordering of the parameters and a proper use of integer or fractional power expansions. In particular, a non-standard application of the MSM is illustrated for the 1:1 resonant case, requiring fractional powers and accounting for the 'arbitrary constants' generally omitted in regular cases.

4. Recently, the problem of passive control of vibrations has been enriched by very peculiar systems, namely the Nonlinear Energy Sinks (NES). NES generally are one DOF oscillators provided with a smaller mass than that of the main structure to be controlled, attached to it by means of a dashpot, which induces small linear damping, as well as an essentially nonlinear spring, which is their distinctive feature. In fact, the essentially nonlinear spring causes lack of linear stiffness in the NES and, on the one hand, allows them to get in resonance with the main structure in a wide spectrum of frequencies, then providing to these devices a greater potentiality than tuned mass dampers; on the other hand, the corresponding nonlinearizable nature of such kind of systems makes the use of standard perturbation methods a difficult task. In particular, it 
was stated in [36], where a NES was applied to a single DOF main oscillator that 'for this type of problem, the standard analytical techniques from nonlinear dynamics (such as the MSM and the standard method of averaging) are not directly applicable, and an alternative approach must be followed.' Fascinated by this challenging problem, Angelo Luongo introduced an innovative way of alternate application of both MSM and harmonic balance for nonlinear systems coupled with NES, giving rise to the so-called Multiple Scale Harmonic Balance Method. It has been first applied to an internally non-resonant multi-DOF system, under harmonic, 1:1 resonant, external force [34]; then, it has been exploited for a two DOF airfoil under wind effect inducing galloping oscillations [35]. Very recently, the method has been extended to continuous structures in direct approach, i.e. applying it to the partial differential equations of an infinite-dimensional system, namely an internally non-resonant string [37].

\section{Elastic buckling and structural analysis}

Immediately after the first studies on cable dynamics, the elastic buckling represents the beginning of his activities on stability issues that are recently bringing fruitful and innovative results even in the context of structural analysis; moreover, starting from buckling researches, significant advances in understanding the localization phenomena in both static and dynamic fields have been made.

\subsection{Elastic buckling}

This group of papers, [38-42], developed in the first decade of his scientific activity, reflects the growing originality of the topics and provides the basis of some ideas spread throughout the subsequent activities. In particular:

1. The interaction of simultaneous buckling modes on the post-buckling behavior of uniformly compressed channels, simply supported at their ends, is investigated [38] on the basis of the general Koiter theory of elastic stability, considering the column as a plate assemblage. The total potential energy is hence written up to third-order terms in order to investigate asymmetric buckling phenomena. The displacement field is described following the classic Vlasov theory where the assumption of rigid cross-sections is removed. A non-traditional finite strip technique is developed through an automatic (and innovative, for the time) procedure of algebraic manipulation. The previous analysis is related to a single interaction between two buckling modes and has been extended in [39] where it is assumed that two or more buckling modes can interact simultaneously, taking into account the initial imperfection effect and analyzing simply supported stiffened angle sections and channels. A simple approximate procedure for solving nonlinear equations related to the evaluation of bifurcated paths is suggested. In the same context, the effect of boundary conditions on multiple interactive buckling in compressed thin-walled members is analyzed [40] pointing out that, under suitable simplifying assumptions, the governing nonlinear equations can be replaced by a linear eigenvalue problems independent of geometric and mechanical properties of the beam, and depending on boundary conditions only. Moreover, the occurrence of a cluster of local buckling modes leads to local deformations concentrated in one or more narrow bands of the beam axis, called 'localization of the buckling patterns.'

2. A significant contribution to the understanding of the nonlinear interaction between several buckling modes in nearly symmetric systems is provided [41], focusing the interest on a perturbation method capable of correctly describing the nonlinear equilibrium paths of perfect and imperfect structures. The method considers the actual system as a perturbation of a properly chosen symmetric one, and does not exhibit the drawbacks of the classic Budiansky's approach where all nonlinearities appear at the same level.

3. Interactive buckling of an elastically restrained truss structure is investigated by proposing an improved version of the Byskov-Hutchinson perturbation analysis [42], which permits avoidance of the iterative procedure related to the load value. The interesting structure under investigation consists of two horizontal beams connected by rigid diagonals, whose out-of-plane displacements are prevented by a continuous distribution of linear springs. When the beams are compressed, three buckling modes, an overall and two local ones, may occur nearly simultaneously and interact in the post-critical range. This model, that Angelo Luongo refined by analyzing localization phenomena (see ahead, Sect. 4.3), inspired other authors in literature, e.g., [43], who referred to it as the 'Luongo's model.' 


\subsection{Structural modeling}

The papers [44-52] are specifically devoted to modeling problems of structural interest; the main original results are summarized in the following.

1. A nonlinear one-dimensional model of a thin-walled beam with generic, non-symmetric open cross-section is developed as an internally constrained three-dimensional continuum [44], subject to axial and shear undeformability. Thanks to cross-section constraints, the warping is expressed in terms of displacements of the shear center by extending the Vlasov theory to the nonlinear field; in particular, the in-plane displacements, which arise as a second-order effect of the out-of-plane classic warping, have been accounted for.

2. A consistent model of a linear, curved, prestressed, no-shear, elastic cable-beam, loaded by wind forces, is formulated [45]. The incremental equilibrium equations around the prestressed state are derived, in which shear forces are condensed. The equations of motion are then greatly simplified by estimating the order of magnitude of all their terms, under the hypotheses of small sag-to-span ratio, order-1 aspect ratio of the (compact) section, characteristic section radius much smaller than length (slender cable), small transversal-to-longitudinal and transversal-to-torsional wave velocity ratios. The model is reformulated in the nonlinear range and nonlinear; reduced equations are derived along the same lines in [46]. These equations are a particular, asymptotic case of more complete models which, besides being composed of very complex equations, suffer of some numerical problems related to the existence of boundary layers, caused by the smallness of the flexural terms (nearly singular equations). Simple discrete nonlinear systems are then derived from the continuous model via standard Galerkin procedures.

3. Innovative approaches are proposed for the cross-sectional analysis in the context of the classic Generalized Beam Theory (GBT), which requires a two-step evaluation procedure, consisting of an initial choice of the vector basis and its successive orthogonalization; they are applicable to generic cross-sections, i.e. open, partially closed and closed ones. The novelty relies in formulating the problem in the spirit of Kantorovich's semi-variational method, evaluating the in-plane deformation modes as the eigenfunctions of a positive-semi-definite auxiliary problem [47]. This latter is chosen as the free dynamic analysis of an unconstrained planar frame, representing the cross-section of the structural member; warping is then evaluated from the post-processing of these in-plane modes, thus reversing the strategy of the classic GBT procedure. Thereafter, a new method identifying a set of conventional modes in a single-step cross-sectional analysis, for any type of cross-section, is proposed [48]. The algorithm differs from that of the classic GBT, and it is based on the definition of a new quadratic functional, whose steady condition leads to a simple eigenvalue problem: in this way, it directly generates the sought orthogonal basis that can be found using a finite-element approach. A new 'complete dynamic approach' is presented in [49], able to evaluate the whole set of conventional and non-conventional modes. This innovative method, referred to as GBT-D, is based on the formulation of two distinct eigenvalue problems, relevant to a segment of thin-walled beam of unitary length, namely (i) a Planar Eigenvalue Problem, governing the in-plane free oscillations of the segment, behaving as an extensible planar frame, and (ii) a Warping Eigenvalue Problem, governing the out-of-plane free oscillation of the segment, behaving as a purely shear beam. Both eigenvalue problems are differential in origin and can be transformed in an algebraic problem applying a suitable discretization to the cross-section middle line. In summary, the basis obtained in this study is formed by: (a) conventional modes (which include in-plane and out-of-plane components); (b) extensional modes (being of purely planar type); and (c) shear modes (being of purely warping type).

4. An asymptotically exact method for static and dynamic analysis of geometrically nonlinear planar frames is described [50], based on the integration of nonlinear equations of inextensible and shear indeformable beams via a perturbation method, under the simplifying assumption that masses are lumped at the beam ends. Goal of this procedure is the numerical evaluation of series expansion coefficients, which describe the response in terms of a control parameter: this is achieved by solving few linear problems (typically three) instead of a large number of nonlinear problems, as required by commonly used iterative methods.

5. A constitutive model for the planar interface of a soft body sliding on a rigid surface is formulated [51], accounting for stick-slip phenomena (caused by friction) and for wear (caused by abrasion). It has been developed in the framework of Damage Mechanics, by introducing gap, isotropic hardening and wear as internal variables of phenomenological type, through the formal analogy between abrasion of a soft body and ductile damage of an elastic-plastic material. Four different sub-models can be further deduced, of no-wearable or wearable type, both with linear or nonlinear isotropic hardening. 
6. A nonlinear model of viscoelastic balloon, interposed between a couple of moving rigid bodies, is proposed [52], modeling the pneumatic structure as a thin, infinitely long cylindrical membrane, prestressed by an internal pressure. The planar problem is addressed with the objectives of formulating a sufficiently simple model, able to give insights the physics of the phenomenon via analytical or semi-analytical solutions, to account for viscoelastic properties of the material, and to consider both the possible flattening and indentation of the balloon, as sequential stages.

\subsection{Localization in statics and dynamics}

Systems that exhibit a great number of nearly simultaneous modes are characterized by buckling patterns in the post-critical range that strongly depend on the load intensity. The deformation can localize in one or more regions of limited size, in contrast to the periodic character of critical modes, giving rise to the so-called localization; it has been addressed in static and dynamic field. The static phenomenon of localization due to interactive buckling problems is first analyzed in [53], with the help of a specific model (the 'Luongo's model,' a 3D beam system consisting of two elastic beams connected by bars which are perfectly rigid inplane and infinitely flexible out-of-plane; the beams are compressed and continuously restrained by elastic springs orthogonal to their plane). This paper demonstrates that, as in the dynamic field, the localization mechanism for local buckling calls for satisfaction of two requisites: (i) the system should have a high modal density, and (ii) it should present a 'structural irregularity' in a broad sense (i.e., a variable geometric stiffness due to the effect of primary bifurcation). Going to analyze localization phenomena in dynamic problems, longitudinal free oscillations of a beam with small axial rigidity, continuously restrained by imperfect elastic springs, are studied in [54], taking into account small imperfections in the spring stiffness in order to obtain the localization of the modal shapes. Systems with single localized defects are first considered, providing physical insights and pointing out that the phenomenon is governed by a turning point mathematical problem. Periodic or nearly periodic imperfections are then analyzed by applying the Floquet theory and, finally, non-periodic imperfections are numerically treated. The asymptotic WKB method proves to be an efficient tool for evaluating the localized eigenfunctions and the associated eigenvalues. The localization phenomenon in linear continuous one-dimensional systems is considered in [55] both in dynamics and buckling. A general perturbation method is developed which generalizes the classic WKB method. It is applied to analyze structures governed by nearly defective system matrices, so their characteristic exponents are highly sensitive to imperfections. Fundamental concepts about localization are introduced, and similarities between dynamics and buckling localization are highlighted. In particular, strong localization of the normal modes is due to turning points in which purely imaginary characteristic exponents assume a nonzero real part; in contrast, if turning points do not occur, only weak localization can exist.

\section{Bifurcation and stability of non-conservative systems}

This is perhaps the most articulated field of activity in which he put to good use many of the experiences gained in previous researches and can be divided into some papers most aimed at methodological issues and some other most devoted to structural applications.

\subsection{Methods}

This section is devoted to briefly resume the key aspects of a class of papers devoted to the development of perturbation methods targeted to the study of bifurcation and stability problems in non-conservative systems. In particular, the topics that can be recognized in these papers concern the investigation of: (a) divergenceHopf bifurcation [56-58], (b) double-zero and multiple-zero bifurcations [59,60], (c) simple Hopf bifurcation [61], multiple (non-defective) Hopf bifurcation [62,63] and multiple (defective) Hopf bifurcation, [64],

(d) theoretical and technical [65,66], and algorithmic [67] aspects in perturbation methods.

Concerning the item (a), the novel focal points are the followings.

1. In [56] the MSM is adapted to study the post-critical behavior of general non-conservative symmetric systems, possibly affected by imperfections, for which divergence and Hopf bifurcations interact. The main contribution given by the procedure illustrated here regards the effectiveness of the MSM with respect 
to the computational burden related to the application of the center manifold reduction. In particular, the paper highlights how it is possible to obtain closed-form expressions for the coefficients of the bifurcation equations of a general system (having two control and one imperfection parameters) in terms of the coefficients of the original system. Finally, the method is applied to a two DOF rigid bar subjected to axial load (Augusti's model) and transversal flow in order to address in detail the critical and post-critical scenarios, for both the perfect and imperfect systems, whose solution is obtained as a perturbation of the perfect system solution.

2. A unified perturbation approach, based on the MSM, is discussed in [57]. This paper gives a key to the understanding of the interactions among static and/or dynamic bifurcations of nonlinear structures. Moreover, a general perturbation algorithm, devoted to the study of multi-parameter, finite-dimensional autonomous system, simultaneously undergoing divergence and two Hopf bifurcations, of non-resonant or resonant type, is built up through the same steps of the static perturbation method; but, differently from this latter, more parameters (not just the load) are considered simultaneously, in order to exhaustively describe the system behavior around the critical point. The method furnishes the bifurcation equations governing the time evolution of amplitudes and phases of the critical interacting modes. The peculiar aspects of dynamical problems compared with static ones are as follows: (i) the amplitudes depend on time; (ii) several time scales are introduced; and (iii) the solvability and bifurcation equations are differential, instead of algebraic. Finally, due to the non-conservativeness of the systems here presented, two sets of right and left eigenvectors are needed by the procedure.

3. In [58], a nonstandard application of the MSM, devoted to analyze the transition from codimension-3 (double-zero/Hopf) to codimension-2 (single-zero/Hopf) bifurcations, occurring in a two DOF, is presented. The bifurcation equations of the system, here obtained, lead to a four-dimensional dynamical system, consisting of a first-order complex equation (as in the Hopf bifurcation) and a second-order real equation (as in the Takens-Bogdanov bifurcation), coupled by mixed terms; it is shown that this system can be reduced to a three-dimensional system, coherently with the codimension of the problem. The method possesses some crucial aspects, namely: (i) fractional power expansions, both for state-variables and time, are used due to the presence of a not-semi-simple double-zero eigenvalue, although the purely imaginary eigenvalues are non-defective; (ii) high-order arbitrary amplitudes are introduced in order to avoid inconsistent results and loss of some terms in the bifurcation equations; and (iii) the reconstitution procedure is not straightforward, since it requires the use of the Schwarz conditions, or, alternatively, $a d$ hoc combinations of the solvability conditions.

Concerning the item (b), the main results can be resumed as below.

1. In [59] different asymptotic techniques, based on perturbations both of the eigenvalue problem and of the characteristic equation, are developed in order to perform the sensitivity analysis of the double-zero eigenvalue and to built up the linear stability diagrams for a general multi-parameter nonconservative system. The analysis sheds light (1) on the existence of a generic (nonsingular) case, for which the double-zero eigenvalue manifests itself at the intersection of a divergence and a Hopf manifold in the parameter space, and (2) on the existence of a non-generic (singular) case for which different bifurcation mechanisms can manifest themselves, such as the double divergence, the double divergence Hopf, and the degenerate Hopf. Moreover, a second-degree equation, uniformly valid around the critical point, is found, thus permitting (i) to capture the essential aspects of the bifurcations' mechanisms, and (ii) to clarify the geometrical meaning of the unfolding parameters, discussed in the literature for the Takens-Bogdanov bifurcation.

2. Multiple-zero bifurcation of a general multi-parameter dynamical system is discussed in [60], which represents an original and important contribution, since its core consists in the exploration of the analogies between sensitivity and bifurcation analysis in defective systems. A MSM algorithm, based on the use of timescales with suitable fractional powers of the perturbation parameter, is developed for a general system in order to obtain the bifurcation equation. The crucial aspect of the algorithm concerns the application of the reconstitution method, which leads to an ordinary differential equation in a unique unknown amplitude, of order equal to the algebraic multiplicity of the zero eigenvalue; this equation, which asymptotically governs the dynamics around the bifurcation, can be seen as a generalization of the Bogdanov-Arnold normal form equation. Finally, by applying the developed algorithm, the nonlinear behavior around the bifurcation of a mechanical system is discussed.

With regard to item (c), the principal achievements can be summarized as detailed in the following. 
1. Nonlinear interaction phenomena among galloping modes of slender structures having narrow band spectra are discussed in [61]. These structures, for which combination resonance phenomena occur, are modeled as nearly periodic systems consisting of weakly coupled beams. By applying the MSM, a system of nonlinear differential equations in the amplitudes and phases of the interactive modes is derived for an n-elements chain. Moreover, numerical results relevant to a two-beam system are presented, also in the case of small imperfections causing asymmetry of the structure. The impressive findings given by this paper are as follows: (i) for the perfect system, the existence of monomodal steady-state (unmodulated) solutions involving the two components of the motion; (ii) for the asymmetric system, the existence of a bimodal solution only; (iii) for critical values of wind velocities, the appearance of stable limit cycles as a result of a Hopf bifurcation.

2. In [62] a two-time version of the Lindstedt-Poincaré Method and the MSM is developed to analyze nonresonant double Hopf bifurcations of a general two-parameter dynamical system. The crucial findings of the paper are as follows: (1) the MSM furnishes amplitude and phase modulation equations identical to normal form equations well known in the literature; (2) the Lindstedt-Poincaré Method can also be considered if only steady-state solutions have to be found; (3) both the methods can be applied to any order to improve the approximation; moreover, they furnish closed-form expressions for the coefficients that could be directly used in applications. It is important to remark that the developed methods are attractive since: (i) they are systematic and consistent; (ii) they are notably aimed to the analysis of large dimensional systems; and (iii) they can be easily extended to analyze higher codimension bifurcation problems, also in the presence of resonance conditions.

3. The paper [63] deals with the study of the post-critical behavior of a general $N$-dimensional system around a 1:2 or 1:3 resonant double Hopf bifurcation. To this end, the MSM is employed in order to obtain, in closed form, the bifurcation equations in terms of the derivatives of the original vector field evaluated at the bifurcation point. The relevant aspects can be resumed in: (i) by truncating the analysis at the thirdorder in both the 1:3 and 1:2 cases, first- or second-order bifurcation equations are obtained; (ii) a mixed polar-Cartesian representation for the amplitudes is used in the paper, in order to obtain a set of three bifurcation equations in standard normal form, in contrast to the usual polar representation which instead leads, in the presented case, to nonstandard form equations; (iii) a self-excited two DOF system is studied, and bifurcation diagrams are built up to give a full representation of the codimension-3 bifurcation.

4. An algorithm based on the MSM, inspired by a formal analogy with the sensitivity analysis of eigenvalues of nilpotent matrices, has been developed in [64], in order to analyze 1:1 resonant multiple Hopf bifurcations in autonomous discrete dynamical systems. When parameters are quasi-statically varied, an arbitrary number of critical eigenvalues simultaneously crosses the imaginary axis. The Jacobian matrix is defective at the bifurcation point so that: (1) only one proper eigenvector exists at the critical point and, as a consequence, the eigenspace must be completed by generalized eigenvectors; and (2) the procedure calls for the use of fractional powers expansions of both state-variables and time, as opposed to integer power expansions used for non-defective bifurcations. The method is specialized for a 1:1 resonant double Hopf bifurcation, and a step-by-step algorithm is presented to evaluate the coefficients of the relevant three real first-order bifurcation equations.

With respect to item (d), the peculiar aspects are resumed in the following.

1. In the paper [65] an exhaustive review, concerning both theoretical and technical aspects of the bifurcation analysis in general, finite-dimensional, autonomous, nonlinear dynamical systems, is addressed. Due to its particular features, dealing with several problems in bifurcation analysis, this paper regards several concepts and methods discussed in this Section; in particular, it is remarkable to notice: (1) the basic concepts of perturbation analysis: (2) low codimension bifurcations in sample systems; (3) mechanical and geometrical aspects in the bifurcation phenomena; (4) sensitivity analysis of the eigenvalues in nondefective and defective cases; (5) integer and fractional power series expansions and reconstitution method in the sensitivity analysis; (6) the MSM for the analysis of multiple bifurcations; (7) the MSM in the case of non-defective and resonant bifurcations; (8) the MSM in the presence of defective bifurcations; and (9) the reconstitution method in the MSM.

2. Paper [66] concerns higher-order MSMs for general multi-parameter mechanical systems. Some important theoretical and technical issues are discussed here, namely: (1) the definition of the codimension of the problem in general systems excited either externally or parametrically; (2) the role of parameters in the perturbation analysis; (3) the number of solvability conditions obtained at each step of the perturbation algorithm; (4) the concept of reduced dynamical system; (5) the critical discussion of four classes of methods, based on the consistency or inconsistency of the approach, and on the completeness or incompleteness 
of the terms retained in the analysis, which are followed in the literature in dealing with reconstituted amplitude equations; and (6) the study of three examples, concerning a static codimension- 1 bifurcation problem, a simultaneous dynamic bifurcation/external resonance problem with codimension- 2 and a free oscillation problem.

3. A numerical perturbation method, based on the sensitivity analysis of eigenvalues, is developed in [67]. The paper focuses on the development of an algorithm able to build up the linear stability diagrams of multi-parameter dynamical systems. It is important to remark that the algorithm developed here is general and, to illustrative purposes only, is applied in the case of codimension-2 bifurcations. Some important issues are remarkable, namely: (1) the algorithm is based on the finding of the codimension-2 bifurcation point and on the building up of the branches emanating from this point; (2) the perturbation method proposed reverses the usual approach, according to which one first looks for codimension- 1 bifurcation loci and, then, follows them by checking, at each step, if a codimension-2 point is encountered; (3) the algorithm is able to furnish the critical combinations of parameters causing multiple bifurcations of static, dynamical, or mixed types.

\subsection{Applications}

The important developments on perturbation methods described in the previous section, specifically addressed to non-conservative systems, have led to numerous applications in subjects of great importance for structural engineering such as cables, tower buildings, and beams.

\subsubsection{Cables}

The common denominator of the papers in this sub-section, [68-74], is the presence of aeroelastic action which leads to problems of fluid-structure interaction known as galloping, usually treated within the quasi-steady approach in the dynamics of iced cables.

Dealing with section models in a linear field, a perturbation method to analytically evaluate the eigensolutions of a two DOF coupled translational galloping for any frequency ratio has been developed in [68]. By introducing the invariants of the total and aerodynamic damping matrices, the eigenvalues can be obtained in a simple and expressive form: the conditions of incipient instability are analyzed in the plane of the aerodynamic damping matrix invariants, where each point is representative of a family of cross-sections with given aerodynamic characteristics. It is noteworthy to observe that Hopf bifurcations can occur as simple bifurcations (classic mono-modal galloping), quasi-simultaneous bifurcations (quasi-bimodal galloping), and double bifurcations (bimodal galloping).

In [69] the nonlinear response of a flexible elastic suspended sagged cable in internal resonance conditions is presented: it is assumed to be at the first "cross-over" point so that its linear frequencies are almost in a 2:1 ratio. Wind forces, acting perpendicular to the plane of the cable, are directly applied to the cable in the normal and bi-normal direction neglecting the effect of its flexibility. In order to analyze coupling phenomena between in-plane and out-of-plane motions, a simple two DOF model is derived via the Galerkin procedure, by considering one modal shape in each plane. Due to mean wind forces, the cable undergoes a static rotation and the equilibrium is set on a inclined plane: this fact produces a modification of aerodynamic forces making galloping a bounded phenomenon, which occurs in a limited range of wind velocities starting from the critical one (differently from what is usually reported in literature). A bifurcation analysis of the amplitude equilibrium path reveals a variegated post-critical behavior, with the presence of both mono-modal (in-plane) and bimodal (two-components) galloping oscillations. A purely planar motion never occurs due to both steady-state forcing terms and nonlinearities. Therefore, geometric nonlinearity modeling is crucial for a proper description of the aeroelastic phenomenon.

Reference [70] makes a step forward, offering a more accurate and simpler description of the equilibrium configuration of the elastic sagged cable, and developing a perturbation approach in the continuous field of the partial derivative nonlinear equations of motion, thus avoiding the introduction of a discrete model. The equations of motion around the equilibrium position are formally identical to the cable equations subject to the sole self-weight except for the use of a fictitious gravity force depending on the mean wind speed. Concerning the solution, when the MSM is directly applied to partial differential equations, it takes into account the contribution of passive modes too, which arise through the origin of secondary modes; these modes are responsible in turns for the spatial alteration of oscillation shapes that are induced by nonlinearities. 
With the objective of analyzing some particular cases highlighted by the scenario of the critical conditions deduced from the linear section model (in particular, the double Hopf bifurcation), the nonlinear post-critical behavior of single taut strings in 1:1 primary internal resonance can be dealt with [71]. By applying the Galerkin method and using the first in-plane and out-of-plane eigenfunction of the linearized problem, a discrete two DOF model is obtained where the mechanical quadratic nonlinearities are absent due to the hypothesis of taut string. The local post-critical behavior is analyzed solving the equations of motion around the bifurcation point. Using the MSM, nearly resonant and non-resonant amplitude equations are drawn in which the existence and stability of coupled, two-mode solutions deserves special attention.

The nonlinear model of cable-beam, described in Sect.4.2, is used in $[72,73]$ to investigate the nonlinear galloping behavior of a suspended cable under internal resonance conditions comparing analytical and numerical approaches. In particular, a cable close to the first crossover point is considered: its first symmetric in-plane mode is in internal resonance of 1:1 type with the first antisymmetric in-plane and out-of-plane modes, and of 2:1 type with the first symmetric out-of-plane mode. The problem is tackled in three different ways. First, the nonlinear integro-partial differential equations are spatially discretized by the finite-difference method and numerically integrated in time. Then, a standard Galerkin method is applied to obtain a few DOF discrete model, whose solutions are drawn both numerically, through direct integrations of equations, and analytically, by using the MSM. Comparisons on a sample case study point out the importance of an extensive analysis of critical conditions and of the internal resonance conditions. In particular, the classic galloping mode of the technical literature (first symmetric in-plane mode) actually becomes unstable for the simultaneous unstabilization of the antisymmetric planar mode, in resonance with it. The three different methods turn out to be complementary for understanding the phenomenon and show a good agreement, from both qualitative and quantitative points of view. The analytical approach seems particularly important to investigate some conditions which are difficult to interpret with sole numerical tools (for instance, unstable regions in fixed-point branches, with slight variations in amplitude). The use of a multimodal approach (like the finite-difference technique) leads to small, quantitative differences with the Galerkin method if the active modes are properly chosen.

Large amplitude vibrations may occur on cables caused by parametric excitation due to small periodic movements of the supported structures. Reference [74] makes a remarkable attempt to study the interaction among three excitation sources (external, parametric, and self-excitation) on a reduced two DOF nonlinear model, able to describe the dynamic behavior of an inclined cable belonging to a cable-stayed bridge. The model of the cable is able to twist, and a sinusoidal vertical motion of given amplitude and frequency is imposed on the lower end, simulating the traffic on the deck. A uniform wind flow, blowing under a yaw angle with respect to the plane of the static configuration of the cable, is also applied; moreover, the presence of a fixed rivulet, breaking the cross-section symmetry, is accounted for. The motion equations are discretized via the Galerkin method, by assuming one in-plane and one out-of-plane modes. The two resulting secondorder, non-homogeneous, time-periodic, ordinary differential equations are coupled, containing quadratic and cubic nonlinearities, both in displacements and velocities. Internal resonance between the two selected modes and external resonance between the forcing and natural frequencies are accounted for. It is shown that, when the motion of the support is small, the galloping is perturbed and islands of non-trivial periodic motions of large amplitude exist, also for velocities smaller than the critical one. When the motion of the support is large, remarkable out-of-plane oscillations occur in no-wind case. Quite surprisingly, such oscillations are drastically reduced in the presence of wind, due to the combined effect of the two sources of excitation. Moreover, the existence of quasi-periodic motions and homoclinic bifurcations has been ascertained.

\subsubsection{Tower buildings}

Wind can induce troublesome and unsafe vibrations in slender structures. In this context, structural modeling and dynamical behavior analysis play a crucial role for design purposes. In particular, in [75] the tower is a linear elastic multistory shear-type frame, modeled through a corresponding homogeneous scheme of continuous shear cantilever, and then reduced to a one DOF system via a Galerkin projection. The structure has been considered under the effect of wind, whose turbulent component is modeled explicitly as a bi-harmonic force giving rise to both parametric and external excitations. This paper has the great merit of clarifying the way in which turbulence can modify the curves of galloping. When the turbulence produces external excitation only, it works as an imperfection, slightly modifying the galloping (perfect) solution, which acts as a backbone for the perturbed (imperfect) states. When the turbulence produces parametric excitation only, the classic galloping curve splits and translates in opposite directions. When the turbulence produces both external and parametric 
excitations, new branches occur, descending from loops of the existing ones; periodic solutions are also found causing quasi-periodic oscillations for the tower. In the same framework, a system constituted by two towers, linked by a nonlinear viscous device at the tip, has been considered [76]. The towers were assumed of different stiffness characteristics, leading to an internally non-resonant system, but with the same critical condition to induce galloping. In this way, when the sole steady part of the wind is applied, the classic unfolding of a non-resonant double Hopf bifurcation is found. The presence of the turbulence, which still works as an imperfection of the system, induces an even more cumbersome dynamics involving periodic, quasi-periodic, mono-modal, and bi-modal solutions.

\subsubsection{Beams}

In this Section, some results, concerning the papers [77-84], which are devoted to apply on beam models the perturbation methods discussed above, are briefly synthesized.

1. In a group of three papers, namely [77-79], the MSM is applied to a one-dimensional continuous cantilevered planar beam, equipped with a lumped viscoelastic device and loaded by a follower force. It has been shown that the beam model here addressed can be considered as a paradigmatic system undergoing all the low codimension bifurcations of mechanical interest. In particular, in [77] the nonlinear, integrodifferential equations of motion are derived expanded up to cubic terms, and, moreover, they are rearranged in an operator form by incorporating the mechanical boundary conditions. The linear stability analysis of the beam is studied, thus highlighting the existence of a rich scenario which reveals divergence, Hopf, and double-zero bifurcations. For these latter, a multiple scale analysis, able to underline the effectiveness of the MSM in obtaining reduced-order models of infinite-dimensional systems, is developed, and preliminary numerical results are illustrated for the double-zero bifurcation. In [78] the main steps of the multiple scale analysis, detailed in [77], are resumed in a more problem-oriented form; moreover, a deep numerical investigation, which leads to several new results, concerning both the linear stability and the post-critical scenario around the three different bifurcation points, is developed. Finally, a deeper parametric analysis, performed in [79], revealed a richer bifurcation scenario, thus including Hopf-divergence and both resonant and non-resonant double Hopf, not discovered in the two previous papers. Then, the bifurcation diagrams, illustrating the system behavior around these critical points of the parameter space, are discussed.

2. References [80] and [81] deal with the stability and the bifurcation of a cantilevered elastic beam (the so-called generalized Beck's column), modeled as a nonlinear Cosserat rod having rectangular crosssection, under the action of a follower tangential force and a bending conservative couple at the free end. In [80], nonlinear, partial integro-differential equations of motion have been derived expanded up to cubic terms in the transversal displacement and torsional angle of the beam. The linear stability analysis of the trivial equilibrium, which reveals the existence of buckling, simple Hopf, and double-zero bifurcation, is developed, and the spectral properties and critical modes of these three instability mechanisms are derived and discussed. In [81], the post-critical behavior of the beam is deeply analyzed investigating the system behavior around bifurcations in the parameter space. In particular, the bifurcation equations for simple buckling (divergence), simple Hopf and double-zero (Takens-Bogdanov) bifurcations are derived by means of the MSM. As main results, it has been found that buckling is of supercritical type, while the simple Hopf bifurcation is of supercritical type far from the double-zero and of subcritical type close to this point, thus entailing that the interaction between the two instability forms leads to an erosion of the mechanical properties of the system.

3. In [82] and [83], the mechanical behavior of a non-conservative nonlinear beam, undergoing divergence, Hopf and double-zero bifurcations, is discussed. The beam model analyzed in these papers is remarkably different with respect to previous ones, since it consists of a cantilevered viscoelastic beam, externally damped and loaded at the tip by follower and dead forces, simultaneously. Preliminary results, concerning both the linear stability and the post-critical behavior around the double-zero bifurcation point, are discussed, in a short form, in [82]. Instead, the derivation of the equations of motion, the study of the stability of the linearized beam, the detailed multiple scale algorithm and a deep analysis in nonlinear regime, can be found in [83]. These papers show that both the linear and the nonlinear scenarios are strongly influenced by damping, since: (a) the position of the double-zero point and the angle of attack between the incident, divergence and Hopf bifurcation loci depend on the damping coefficients, and the property of the undamped system is recovered only for evanescent external damping, thus revealing new aspects of the 'destabilization paradox'; (b) the nonlinear scenario, around the double-zero, is strongly affected by 
the external damping since the static bifurcation is supercritical when it is small, but becomes subcritical when it is large.

4. The problem of Nicolai concerning the dynamic stability of a continuous elastic cantilevered rod, having different principal moments of inertia and loaded by an axial compressive force and a twisting tangential torque, is addressed in [84]. New governing equations and boundary conditions, which take into account also for the pre-twisting of the rod due to the torque, are derived. The stability region in the threedimensional space of parameters, namely the axial load, the tangential torque and the ratio of principal moments of inertia, is found via a perturbation analysis; moreover, the singular point on the stability boundary at the critical Euler force is recognized and investigated in detail. Finally, the stability region in the case of an elliptic cross-section is found numerically, and it is shown that the numerical results agree with the analytical formulas of the asymptotic analysis.

\section{New advances and research perspectives}

The scientific personality of Professor Angelo Luongo is characterized by a tireless push to creativity. Despite the very difficult moment that the Italian Academia is experiencing, he managed to lead a group of scientific researchers toward relevant results in both theoretical and applied mechanics. The brilliant achievements which he got as a research leader and guidance assume even greater importance in the perspective of the enormous difficulties which he faced. However, he stubbornly pursued originality and has been very demanding to his pupils and co-workers managing, until this was possible, to get their best out of them.

Angelo Luongo is a Maestro and a true Professor: he has fully dedicated his life to the advancement of science, in the field in which he cultivated his competences. In order to promote international links between researchers on mathematical modeling for engineering, a topic of fundamental importance in both science and technology, he is staunchly committed to found an International Research Centre on Mathematics and Mechanics of Complex Systems-M\&MoCS (the M\&MoCS center is described in the website: http://memocs. univaq.it/?lang=en). In this center, scientists from all over the world cooperate in order to find and develop new research streams, and to push younger scientists to contribute to the advancement of science. In the following, we sketch some of the research projects which are animated by professor Luongo in the framework of the activities of the M\&MoCS Centre.

\subsection{Dynamic behavior, instabilities and wrinkling in (non)linear complex microstructured systems}

A paradigmatic example of complex mechanical system, constituted at a smaller scale by underlying microstructures, is given by fiber reinforcements: they play a crucial role in the modern technology of composite materials whose importance in (aeronautical) engineering cannot be underestimated. In these reinforcements, woven or nonwoven long fibers are connected one to the others by means of complex geometrical patterns. Each of these fibers is subjected to bending deformation, and, therefore, a non-negligible amount of deformation energy may be stored in this form. Second-gradient continuum models have been proposed in order to effectively predict the biaxial test for rectangular specimen constituted by some particular fiber reinforcements (see [85]). Also relevant is wrinkling phenomena which are observed in static deformation tests, to be associated with clear phenomena of loss of stability. Some models of these fabrics involve the introduction of suitable microscopic networks of structural elements interconnected via various kind of constraints. In other words, the microstructure of the fabric is modeled by means of networks of (Euler or Timoshenko) beams (e.g., [86]): the macroscopic behavior of the fabric is being determined by means of suitable homogenization procedures. These procedures may also produce continuous models (see, e.g., $[87,88]$ ) where the microscopic microstructure is accounted for through constitutive anisotropy or constrained kinematics. Another relevant example is given by solids with interconnected pores, saturated, or partially saturated by compressible fluids (see, e.g., [89-92]).

All macroscopic (continuous) models to be used in this context must belong to the class of generalized continua (see, e.g., [93-96]). The main problem to be confronted when modeling with continua fabrics having a complex microstructure consists in the determination of macroscopic constitutive equations in terms of the microscopic mechanical and geometrical features of considered mechanical systems. This problem is usually heuristically approached by means of some perturbation techniques, by considering 'small' perturbative parameters (see, e.g., [97,98]): when the form of the perturbative series for all involved fields is suitably 
assumed, one gets well-grounded conjectures for the macroscopic continuum model to be used. Only in a subsequent step, mathematically rigorous results (as those presented in $[99,100]$ ) can be proven. Therefore, the heuristic 'art of perturbative expansion,' in which Angelo Luongo is a recognized master in all the world, can be an important guide toward mathematical discovery.

A project to be developed in the framework of the activity of M\&MoCS, under the coordination and guidance of Professor Angelo Luongo, consists in the application of perturbation techniques to the study of beam networks exhibiting a complex geometry and high contrast in mechanical properties in order to supply those conjectural 'target' continuum theories for which, more likely, homogenization results could be proven. In addition to possible perturbation approaches, numerical methods play a relevant role both for the study of obtained macroscopic models and also to determine, via homogenization techniques, the correct macroscopic constitutive equations. Therefore, in order to investigate the dynamic behavior (see, e.g., [92,101]), or the onset of instabilities (some of which can determine wrinkling, see, e.g., [102]), one has to be able to formulate suitably effective numerical codes (see, e.g., [103-109] developed in similar context). These codes will either be able to effectively solve problems involving higher space derivatives of kinematic fields, or to supply the solution of prototype problems on periodicity cells (see, e.g., $[113,114]$ ). It has to be remarked that one of the most relevant phenomenon occurring in the considered microstructures consists in the concentration of compression (or traction) of strain or stress along preferential curves: in this context, it will be useful to consider the studies presented in [85] or in [110].

Along this research line, a notable effort has been recently undertaken at M\&MoCS to investigate novel models of "cellular solids", particularly Aluminum Foam Structures (AFS). The AFS are fascinating and innovative engineering materials for primary applications in sustainable lightweight transportation (automotive, aeronautical, aerospace, and maritime), but also they offer appealing possibilities to create "smart" and customizable structural platforms that can incorporate electronics and nanotechnologies (e.g., sensing, communication, and damage healing). However, the behavior and damage tolerance of AFS components depend on (mesoscale and micro-scale) wrinkling phenomena of their randomly textured thin-walls members, that needs to be accounted for in the effective macroscale continuum model, which eludes simplistic modeling and demands complex numerical models. Joining AFS components by welding (see preliminary experimental observations in $[111,112])$ is also a modeling challenges for AFS-based structures which need to be schematized as effective continua filled with discontinuous materials interfaces in order to capture their correct static and dynamic response.

\subsection{Bifurcation phenomena in remodeling and growth of reconstructed tissues}

Recently, an increasing attention has been attracted by phenomena which govern the growth of living tissues and, in particular, the reconstructed bones (see, e.g., $[115,116])$. These phenomena have a relevant mechanical content, and it seems that they can be described by means of suitable mathematical models having a similar structure as those used in disparate fields, for instance, in plasticity. However, in the considered phenomena, a bio-mechanical signal triggers the biological response of the tissue, thus inducing, when appropriate, an increase in the mechanical stiffness of the tissue itself.

Remodeling and reconstruction are intrinsically evolutionary processes whose development is determined, in a critical way, by many parameters. In a recent workshop held at the M\&MoCS center, concerning the critical role of applied external load, Prof. Luongo recognized as a double Hopf bifurcation the phenomenon which has been observed in bone tissue reconstruction. When this load is under a given threshold (see, e.g., [117-119]), then the reconstruction process cannot be completed, and there is a cavity formation; in other cases, either the bone is completely reconstructed (and the scaffold material is totally removed, which is the most desirable possibility), or the bioresorbable material remains trapped inside the reconstructed bone, which makes the final tissue weaker than what is desired. We expect that a careful study of the strongly nonlinear evolution equations which govern the proposed models by using MSM may supply a guidance to interesting engineering optimization problems.

\subsection{Control and stability in innovative systems}

The deep knowledge of Prof. Luongo on perturbation methods can be very fruitfully applied in the field of vibration and energy control. We have already talked about the fact that Angelo Luongo was the first to propose 
an analytical solution for nonlinear systems coupled with NES (Sect.3.3), contradicting the impossibility of finding a perturbation solution asserted by most scientists. The basic concept of 'structural modification' is the following: starting from a structure having a specific function (master structure), one has to add to it a secondary one (slave structure) in order to (i) do not modify the required design functions of the master structure, and (ii) to direct mechanical energy in the slave structure, where it is dissipated or somehow used, in a useful, or at least non-harmful, way. Systems capable of improve the performance of (master) structural modes through targeted energy transfer (e.g., [120,121]) or energy sharing mechanism (e.g., [122-124]) have had convincing applications. The considered slave structures have been considered to be nonlinear or also intrinsically linear with results in vibration control which seem to be promising in both cases.

In the more general class of multi-physics metamaterials, one can consider piezoelectro-mechanical structures as a kind of modified structure. In these cases, the slave structure is given by the electrical waveguides coupled to structural elements via suitably interconnected arrays of piezoelectric actuators (see, e.g., [125]). The effectiveness of such devises as vibration controllers has been proven both theoretically and experimentally (e.g., [126-128]). However, the issue of the stability efficiency of such devices under nonconservative positional forces has been recently highlighted by Prof. Luongo, and needs to be investigated and ascertained. The papers $[129,130]$ present some preliminary results concerning this innovative aspect.

It has to be remarked that modifying a structure, a material or a mechanical system in order to optimize some of their performances could have a detrimental effect in their behavior when other different features are considered. A remarkable example is represented by scale effects on materials properties in nanotechnology, where the mechanical (elastic and inelastic) properties of nanomaterials, such as nanowires and nanoparticles, change dramatically from bulk properties used in conventional structural engineering, and typically scale with component size according to power laws. Starting with the fundamental framework reported in literature (e.g., [131-133]), Professor Luongo championed a project awarded by the Italian Ministry of Education, Universities and Research (MIUR) to investigate the strengthening and stiffening mechanical size effects in zinc-oxide nanowires (which become stronger and stiffer than bulk) and their functional implications on piezoelectric performance and buckling resistance of innovative nanopiezogenerators for energy harvesting $[134,135]$. To achieve these goals, this ongoing project at M\&MoCS pursues a multi-disciplinary approach encompassing advanced nanoscale experiments with complex continuum multi-physics modeling to yield new design principles for the engineering of piezoelectric MicroElectroMechanical and NanoElectroMechanical Systems at large.

\subsection{Localization problems in solids and structures}

The issue of localization has received significant attention from the research activity of Prof. Luongo, as evidenced by the papers [52-55] described in the previous Sects. 4.2 and 4.3. This problem is very relevant in solid and structural mechanics, and the phenomenon of deformation and/or stress concentration in relatively narrow parts of deformable bodies has been observed in various interesting situations. Its mathematical description represents an important challenge for mathematical physics. Relatively few results in this subject are available in the literature: This is mainly due to the fact that a comprehensive conceptual framework able to encompass such complex phenomena is still being constructed by developing so-called theories of generalized continua. The mathematical structure of this problem is similar to the so-called 'Free Moving Boundary Problems.' A region in the reference configuration must be found where deformation or stress undergoes very sharp variations, experiencing relatively high gradients. The possibility of describing a whole and large range of physical states of considered deformable bodies by means of a unique constitutive equation is essential if one wants to establish a mathematical model which is able to forecast the localization of the aforementioned regions. In this context, the general framework can be found in the papers [95,96]: perturbation techniques able to determine boundary layers by means of inner and outer expansions are needed to perform qualitative studies on this phenomenon.

In particular, the issue of the propagation of sharp interfaces and fronts in continua and structures is of great interest to many scientists belonging to the center M\&MoCS, and it has been the subject of numerous meetings chaired by Prof. Luongo thanks to its expertize in localization problems. For instance, quasi-static or dynamic non-material interfaces are able to model the martensitic type phase transitions in solids such as shape memory alloys (see, e.g., [136-138]), where one can find solutions of mono-dimensional problems. Motivated by experimental observations of phase transitions in thin-walled structures (see, e.g., [139-141]), the behavior of thin-walled structural elements made of materials undergoing phase transitions within the nonlinear shell 
theory was considered in [142-145]. Unlike to three-dimensional models of elasticity and plasticity of solids with phase interfacial zones (e.g., [146-148]), the bi-dimensional models based on the shell and plate theory are supplying a powerful tool for decreasing the computational effort needed to design complex thin structures undergoing phase transitions. Another example of sharp interfaces is represented by the strain localization phenomena observed in cellular materials such as polymer and metal foams (see, e.g., [149-153]). For both phase interfaces and strain localization phenomena, the crucial point is the proper formulation of a kinetic equation describing the propagation of the interface. The kinetic relation links the driving force acting on a phase interface with its velocity. Various forms of kinetic equations are extensively discussed in the literature (see, e.g., $[136,137,144,145,154,155])$ : they can be considered as an additional constitutive relation that is necessary for the complete description of the interface motion. There are two possibilities to introduce a kinetic relation: from "first principles" considering discrete systems and its continual counterparts (e.g., [153-155]), or using a phenomenological approach which postulates kinetic equations as additional constitutive relations consistent with the thermodynamics (e.g., [136,137,145]).

In this general framework, problems related with action of a moving load or moving mass along strings, beams, and other structures can be placed, even if their mathematical description does not require the presence of kinetic equations as additional constitutive relations. Recently, as part of M\&MoCS activities, a research attempt on this topic has been started under the supervision of Prof. Luongo focusing, in particular, on the mechanics of moving masses and loads along deformable strings. Although a huge literature has been developed in the field, many open problems seem to wait for a solution. We refer to [156] for an overview of some of them. Here, we limit ourselves to report some aspect of the involved phenomena which need to be more precisely modeled and more deeply understood: (i) the role of geometric nonlinearities in the string deformation, especially in the vicinity of the endpoint of the considered string, (ii) the effect of deformability of the constraints limiting the kinematics of the deformable string, (iii) the effect of the finite dimensions of the moving mass, and (iv) the role of material nonlinearities in the behavior of the deformable string. All these effects need to be investigated by means of perturbation methods such as MSM in order to get a quantitative and qualitative analysis of their relative relevance.

Acknowledgments This work was supported by the Italian Ministry of Education, Universities and Research (MIUR) through the PRIN co-financed program 'Dynamics, Stability and Control of Flexible Structures' (Grant Number No. 2010MBJK5B).

\section{Appendix}

Presentation of the Plenary Lecture 'On the Use of the Multiple Scales Method in Solving Difficult Bifurcation Problems' held by Professor Angelo Luongo at the 4th Canadian Conference on Nonlinear Solid Mechanics (CanCNSM2013), Thursday July 25th, 2013, Montreal, QC, Canada (read by Professor Francesco dell'Isola).

'It is a privilege to be called to present to this audience Professor Angelo Luongo. It could be easy to limit my presentation to a list of numbers: papers published, papers reviewed in Mathematical Reviews (because, you know, even if he pretends to be an 'applied engineer' actually Angelo, by definition, is a mathematician as he authored more than 20 papers reviewed in Math Rev). I could continue by counting his papers listed in Scopus or in the Web of Science or in Google Scholar, and by counting citations which his papers obtained. I could also count the years in which he served as Pro Rettore Vicario (Vice-President) of L'Aquila University, as Department Head or as Director of Research Centers. I could also list a huge amount of research grants obtained by different sources. But this compulsive, obsessive disease which obliges us to count instead of judging is leading us to miss the true scientific content of considered papers and, in this case, the true scientific personality which we want to highlight. Of course, to formulate opinions is risky; to explicitly state that somebody is not or, on the contrary, actually is creative implies an engagement which is personal and is involving the risk of being wrong.'

'After having known him well I dare to say that Angelo Luongo indeed is an original and creative mathematician and mechanician. He introduced in Italy the perturbative methods needed to study elastic buckling and non-conservative problems. He applied them in an independent and novel way to a variety of problems. His opinion in the field is authoritative, and he is the leading personality in the field in Italy, if not in the whole World. He formed many younger scientist and deeply influenced (and scientifically supported) many elder ones. He is a gifted manager: as a department head and pro-rector, he showed his integrity and honesty by always favoring the interest of the academic institution to his own; in particular, he never sacrificed the interest of the institution with actions aimed to be re-elected or to be elected to a higher office. He is a true academician 
(un vero Professore); he never accepted any commercial or professional involvement and dedicated all his forces to serve his vision of progress of science and knowledge. He is the person who strongly wanted the creation of the International Research Center M\&MoCS which is editing a Journal, is organizing or sponsoring qualified conferences like this one and, more important, is supporting really talented young researchers.'

'I believe that he also is an outstanding teacher and I am sure you will gain some deeper insight into multiple scale methods and their application to bifurcation problems by hearing his lecture. In conclusion-this is my own personal opinion-Angelo Luongo is a beautiful example of scholar dedicated to the progress of science as those monks who, by copying ancient and precious books, allowed the wonderful Renaissance of knowledge after Middle Age.'

\section{References}

1. Luongo, A.: Eigensolutions sensitivity for nonsymmetric matrices with repeated eigenvalues. AIAA J. 31(7), 13211328 (1993)

2. Luongo, A.: Free vibrations and sensitivity analysis of a defective two degree-of-freedom system. AIAA J. 33(1), 120$127(1995)$

3. Luongo, A.: Eigensolutions of perturbed nearly defective matrices. J. Sound Vib. 185(3), 377-395 (1995)

4. Luongo, A.: Perturbation methods for nonlinear autonomous discrete-time dynamical systems. Nonlinear Dyn. 10(4), 317331 (1996)

5. Luongo, A., Di Egidio, A., Paolone, A.: Computational problems in multiple scale analysis. In: Recent Research Developments in Sound and Vibration, vol. 2, Transworld Research Network, Kerala, India, pp. 1-31 (2003), ISBN:81-7736-186-4

6. Luongo, A., Di Egidio, A., Paolone, A.: Qualitative analysis of classes of motion for multiresonant systems II. A geometrical method. Acta Mech. 174(1-2), 109-124 (2005)

7. Luongo, A., Di Egidio, A., Paolone, A.: On the proper form of the amplitude modulation equations for resonant systems. Nonlinear Dyn. 27(3), 237-254 (2002)

8. Luongo, A., Di Egidio, A., Paolone, A.: Qualitative analysis of classes of motion for multiresonant systems I. An algebraic method. Acta Mech. 174(1-2), 91-107 (2005)

9. Rega, G., Luongo, A.: Natural vibrations of suspended cables with flexible supports. Comput. Struct. 12(1), 65-75 (1980)

10. Luongo, A., Rega, G.: Discussion of "Free vibration of parabolic cables" by Anestis S. Veletsos and George R. Darbre (February, 1983). J. Struct. Eng. 110(6), 1430-1431 (1984)

11. Romeo, F., Luongo, A.: Invariant representation of propagation properties for bi-coupled periodic structures. J. Sound Vib. 257(5), 869-886 (2002)

12. Romeo, F., Luongo, A.: Vibration reduction in piecewise bi-coupled periodic structures. J. Sound Vib. 268(3), 601$615(2003)$

13. Luongo, A., Romeo, F.: Real wave vectors for dynamic analysis of periodic structures. J. Sound Vib. 279(1-2), 309$325(2005)$

14. Luongo, A., Rega, G., Vestroni, F.: Monofrequent oscillations of a non-linear model of a suspended cable. J. Sound Vib. 82(2), 247-259 (1982)

15. Luongo, A., Rega, G., Vestroni, F.: Planar non-linear free vibrations of an elastic cable. Int. J. Nonlinear Mech. 19(1), 39_ $52(1984)$

16. Luongo, A., Rega, G., Vestroni, F.: On large-amplitude vibrations of cables. J. Sound Vib. 116(3), 573-575 (1987)

17. Luongo, A., Rega, G., Vestroni, F.: On nonlinear dynamics of planar shear indeformable beams. J. Appl. Mech. Trans. ASME 53(3), 619-624 (1986)

18. Luongo, A., Rega, R., Vestroni, F.: Non-resonant non-planar free motions of inextensional non-compact beams. J. Sound Vib. 134(1), 73-86 (1989)

19. Di Egidio, A., Luongo, A., Vestroni, F.: A non-linear model for the dynamics of open cross-section thin-walled beams-part II: forced motion. Int. J. Nonlinear Mech. 38(7), 1083-1094 (2003)

20. Pasca, M., Pignataro, M., Luongo, A.: Three-dimensional vibrations of tethered satellite systems. J. Guid. Control Dyn. 14(2), 312-320 (1991)

21. Luongo, A., Vestroni, F.: Non-linear free periodic oscillations of a tethered satellite system. J. Sound Vib. 175(3), 299315 (1994)

22. Luongo, A., Vestroni, F.: Bifurcations and stability of amplitude modulated planar oscillations of an orbiting string with internal resonances. Nonlinear Dyn. 9(3), 305-325 (1996)

23. Di Egidio, A., Luongo, A., Vestroni, F.: Nonstationary nonplanar free motions of an orbiting string with multiple internal resonances. Meccanica 31(3), 363-381 (1996)

24. Vasta, M., Luongo, A.: Dynamic analysis of linear and nonlinear oscillations of a beam under axial and transversal random poisson pulses. Nonlinear Dyn. 36(2-4), 421-435 (2004)

25. Luongo, A., Romeo, F.: A transfer-matrix-perturbation approach to the dynamics of chains of nonlinear sliding beams. J. Vib. Acoust. Trans. ASME 128(2), 190-196 (2006)

26. Vestroni, F., Luongo, A., Paolone, A.: A perturbation method for evaluating nonlinear normal modes of a piecewise linear two-degrees-of-freedom system. Nonlinear Dyn. 54(4), 379-393 (2008)

27. Belyakov, A., Seyranian, A., Luongo, A.: Dynamics of the pendulum with periodically varying length. Physica D Nonlinear Phenomena 238(16), 1589-1597 (2009)

28. Vestroni, F., Luongo, A., Pasca, M.: Stability and control of transversal oscillations of a tethered satellite system. Appl. Math. Comput. 70(2-3), 343-360 (1995) 
29. Pasca, M., Vestroni, F., Luongo, A.: Stability and bifurcations of transversal motions of an orbiting string with a longitudinal control force. ZAMM Zeitschrift Fur Angewandte Mathematik Und Mechanik 76(SUPPL. 4), 337-340 (1996)

30. Gattulli, V., Di Fabio, F., Luongo, A.: Simple and double Hopf bifurcations in aeroelastic oscillators with tuned mass dampers. J. Franklin Inst. 338(2-3), 187-201 (2001)

31. Gattulli, V., Di Fabio, F., Luongo, A.: One to one resonant double Hopf bifurcation in aeroelastic oscillators with tuned mass dampers. J. Sound Vib. 262(2), 201-217 (2003)

32. Gattulli, V., Di Fabio, F., Luongo, A.: Nonlinear tuned mass damper for self-excited oscillations. Wind Struct. 7(4), 251264 (2004)

33. Arkhipova, I., Luongo, A., Seyranian, A.: Vibrational stabilization of the upright statically unstable position of a double pendulum. J. Sound Vib. 331(2), 457-469 (2012)

34. Luongo, A., Zulli, D.: Dynamic analysis of externally excited NES-controlled systems via a mixed multiple scale/harmonic balance algorithm. Nonlinear Dyn. 70(3), 2049-2061 (2012)

35. Luongo, A., Zulli, D.: Aeroelastic instability analysis of NES-controlled systems via a mixed multiple scale/harmonic balance method. J. Vib. Control 20(13), 1985-1998 (2014)

36. Jiang, X., McFarland, D., Bergman, L., Vakakis, A.: Steady state passive nonlinear energy pumping in coupled oscillators: theoretical and experimental results. Nonlinear Dyn. 33, 87-102 (2003)

37. Zulli, D., Luongo, A.: Nonlinear energy sink to control vibrations of an internally nonresonant elastic string. Meccanica OnlineFirst. doi:10.1007/s11012-014-0057-0 (07 Oct 2014)

38. Pignataro, M., Luongo, A., Rizzi, N.: On the effect of the local overall interaction on the postbuckling of uniformly compressed channels. Thin-Walled Struct. 3(4), 293-321 (1985)

39. Pignataro, M., Luongo, A.: Asymmetric interactive buckling of thin-walled columns with initial imperfections. Thin-Walled Struct. 5(5), 365-382 (1987)

40. Luongo, A., Pignataro, M.: Multiple interaction and localization phenomena in the postbuckling of compressed thin-walled members. AIAA J. 26(11), 1395-1402 (1988)

41. Luongo, A., Plgnataro, M.: On the perturbation analysis of interactive buckling in nearly symmetric structures. Int. J. Solids Struct. 29(6), 721-733 (1992)

42. Pignataro, M., Luongo, A.: Interactive buckling of an elastically restrained truss structure. Thin-Walled Struct. 19(24), 197-210 (1994)

43. Coman, C.: Author's response to Dr.Wadee and Yiatros' discussion of my paper: "On interactive buckling in a sandwich structure" (ZAMP, 61:565-577, 2010). Z. Angew. Math. Phys. ZAMP 62(5), 957-960 (2011)

44. Di Egidio, A., Luongo, A., Vestroni, F.: A non-linear model for the dynamics of open cross-section thin-walled beams-Part I: formulation. Int. J. Nonlinear Mech. 38(7), 1067-1081 (2003)

45. Luongo, A., Zulli, D., Piccardo, G.: A linear curved-beam model for the analysis of galloping in suspended cables. J. Mech. Mater. Struct. 2(4), 675-694 (2007)

46. Luongo, A., Zulli, D., Piccardo, G.: On the effect of twist angle on nonlinear galloping of suspended cables. Comput. Struct. 87(15-16), 1003-1014 (2009)

47. Ranzi, G., Luongo, A.: A new approach for thin-walled member analysis in the framework of GBT. Thin-Walled Struct. 49(11), 1404-1414 (2011)

48. Piccardo, G., Ranzi, G., Luongo, A.: A direct approach for the evaluation of the conventional modes within the GBT formulation. Thin-Walled Struct. 74, 133-145 (2014)

49. Piccardo, G., Ranzi, G., Luongo, A.: A complete dynamic approach to the Generalized Beam Theory cross-section analysis including extension and shear modes. Math. Mech. Solids. 19(8), 900-924 (2014)

50. Contento, A., Luongo, A.: Static and dynamic consistent perturbation analysis for nonlinear inextensible planar frames. Comput. Struct. 123, 79-92 (2013)

51. D'Annibale, F., Luongo, A.: A damage constitutive model for sliding friction coupled to wear. Contin. Mech. Thermodyn. 25(2-4), 503-522 (2013)

52. De Simone, A., Luongo, A.: Nonlinear viscoelastic analysis of a cylindrical balloon squeezed between two rigid moving plates. Int. J. Solids Struct. 50(14-15), 2213-2223 (2013)

53. Luongo, A.: On the amplitude modulation and localization phenomena in interactive buckling problems. Int. J. Solids Struct. 27(15), 1943-1954 (1991)

54. Luongo, A.: Mode localization by structural imperfections in one-dimensional continuous systems. J. Sound Vib. 155(2), 249-271 (1992)

55. Luongo, A.: Mode localization in dynamics and buckling of linear imperfect continuous structures. Nonlinear Dyn. 25(13), 133-156 (2001)

56. Luongo, A., Paolone, A.: Multiple scale analysis for divergence-Hopf bifurcation of imperfect symmetric systems. J. Sound Vib. 218(3), 527-539 (1998)

57. Luongo, A.: A unified perturbation approach to static/dynamic coupled instabilities of nonlinear structures. Thin-Walled Struct. 48(10-11), 744-751 (2010)

58. Luongo, A., Zulli, D.: A paradigmatic system to study the transition from zero/Hopf to double-zero/Hopf bifurcation. Nonlinear Dyn. 70(1), 111-124 (2012)

59. Luongo, A., Paolone, A., Di Egidio, A.: Sensitivities and linear stability analysis around a double-zero eigenvalue. AIAA J. 38(4), 702-710 (2000)

60. Luongo, A., Di Egidio, A., Paolone, A.: Multiple-timescale analysis for bifurcation from a multiple-zero eigenvalue. AIAA J. 41(6), 1143-1150 (2003)

61. Luongo, A., Di Fabio, F.: Multimodal galloping of dense spectra structures. J. Wind Eng. Ind. Aerodyn. 48(2-3), 163174 (1993)

62. Luongo, A., Paolone, A.: Perturbation methods for bifurcation analysis from multiple nonresonant complex eigenvalues. Nonlinear Dyn. 14(3), 193-210 (1997) 
63. Luongo, A., Paolone, A., Di Egidio, A.: Multiple timescales analysis for 1:2 and 1:3 resonant Hopf bifurcations. Nonlinear Dyn. 34(3-4 SPEC. ISS.), 269-291 (2003)

64. Luongo, A., Di Egidio, A., Paolone, A.: Multiscale analysis of defective multiple-Hopf bifurcations. Comput. Struct. 82(3132), 2705-2722 (2004)

65. Luongo, A., Di Egidio, A., Paolone, A.: Multiple scale bifurcation analysis for finite-dimensional autonomous systems. In: Recent research developments in sound and vibration, Vol. 1. Transworld Research Network, Kerala, India, pp. 161-201. (2002) ISBN:81-7895-031-6

66. Luongo, A., Paolone, A.: On the reconstitution problem in the multiple time-scale method. Nonlinear Dyn. 19(2), 133$156(1999)$

67. Luongo, A., D'Annibale, F.: Linear stability analysis of multiparameter dynamical systems via a numerical-perturbation approach. AIAA J. 49(9), 2047-2056 (2011)

68. Luongo, A., Piccardo, G.: Linear instability mechanisms for coupled translational galloping. J. Sound Vib. 288(4-5), 1027$1047(2005)$

69. Luongo, A., Piccardo, G.: Non-linear galloping of sagged cables in 1:2 internal resonance. J. Sound Vib. 214(5), 915$936(1998)$

70. Luongo, A., Piccardo, G.: A continuous approach to the aeroelastic stability of suspended cables in 1:2 internal resonance. JVC/J. Vib. Control 14(1-2), 135-157 (2008)

71. Luongo, A., Paolone, A., Piccardo, G.: Postcritical behavior of cables undergoing two simultaneous galloping modes. Meccanica 33(3), 229-242 (1998)

72. Luongo, A., Zulli, D., Piccardo, G.: Analytical and numerical approaches to nonlinear galloping of internally resonant suspended cables. J. Sound Vib. 315(3), 375-393 (2008)

73. Luongo, A., Zulli, D., Piccardo, G.: Erratum to "analytical and numerical approaches to nonlinear galloping of internallyresonant suspended cables” J. Sound Vib. 315(3): 375-393 (doi:10.1016/j.jsv.2008.03.067). J. Sound Vib. 317(35), 1059 (2008)

74. Luongo, A., Zulli, D.: Dynamic instability of inclined cables under combined wind flow and support motion. Nonlinear Dyn. 67(1), 71-87 (2012)

75. Luongo, A., Zulli, D.: Parametric, external and self-excitation of a tower under turbulent wind flow. J. Sound Vib. 330(13), 3057-3069 (2011)

76. Zulli, D., Luongo, A.: Bifurcation and stability of a two-tower system under wind-induced parametric, external and selfexcitation. J. Sound Vib. 331(2), 365-383 (2012)

77. Luongo, A., Di Egidio, A.: Bifurcation equations through multiple-scales analysis for a continuous model of a planar beam. Nonlinear Dyn. 41(1-3), 171-190 (2005)

78. Luongo, A., Egidio, A.: Divergence, Hopf and double-zero bifurcations of a nonlinear planar beam. Comput. Struct. 84(2425), 1596-1605 (2006)

79. Di Egidio, A., Luongo, A., Paolone, A.: Linear and non-linear interactions between static and dynamic bifurcations of damped planar beams. Int. J. Nonlinear Mech. 42(1), 88-98 (2007)

80. Paolone, A., Vasta, M., Luongo, A.: Flexural-torsional bifurcations of a cantilever beam under potential and circulatory forces I: non-linear model and stability analysis. Int. J. Nonlinear Mech. 41(4), 586-594 (2006)

81. Paolone, A., Vasta, M., Luongo, A.: Flexural-torsional bifurcations of a cantilever beam under potential and circulatory forces II. Post-critical analysis. Int. J. Nonlinear Mech. 41(4), 595-604 (2006)

82. Luongo, A., D'Annibale, F.: Bifurcation analysis of damped visco-elastic planar beams under simultaneous gravitational and follower forces. Int. J. Modern Phys. B 26(25), 1246015-1-1246015-6 (2012)

83. Luongo, A., D'Annibale, F.: Double zero bifurcation of non-linear viscoelastic beams under conservative and nonconservative loads. Int. J. Nonlinear Mech. 55, 128-139 (2013)

84. Seyranian, A., Di Egidio, A., Contento, A., Luongo, A.: Solution to the problem of Nicolai. J. Sound Vib. 333(7), 19321944 (2014)

85. Ferretti, M., Madeo, A., dell'Isola, F., Boisse, P.: Modeling the onset of shear boundary layers in fibrous composite reinforcements by second-gradient theory. Z. Angew. Math. Phys. ZAMP 65(3), 587-612 (2014)

86. Turco, E.: Load distribution modelling for pin-jointed trusses by an inverse approach. Comput. Methods Appl. Mech. Eng. 165(1-4), 291-306 (1998)

87. Federico, S., Grillo, A.: Elasticity and permeability of porous fibre-reinforced materials under large deformations. Mech. Mater. 44, 58-71 (2012)

88. Federico, S., Grillo, A., Wittum, G.: Considerations on incompressibility in linear elasticity. Nuovo Cimento Della Societa Italiana di Fisica C 32(1), 81-87 (2009)

89. Sciarra, G., dell'Isola, F., Coussy, O.: Second gradient poromechanics. Int. J. Solids Struct. 44(20), 6607-6629 (2007)

90. Madeo, A., Gavrilyuk, S.: Propagation of acoustic waves in porous media and their reflection and transmission at a pure-fluid/porous-medium permeable interface. Eur. J. Mech. A/Solids 29(5), 897-910 (2010)

91. Madeo, A., dell' Isola, F., Darve, F.: A continuum model for deformable, second gradient porous media partially saturated with compressible fluids. J. Mech. Phys. Solids 61(11), 2196-2211 (2013)

92. Rosi, G., Madeo, A., Guyader, J.-L.: Switch between fast and slow biot compression waves induced by "second gradient microstructure" at material discontinuity surfaces in porous media. Int. J. Solids Struct. 50(10), 1721-1746 (2013)

93. Steigmann, D.: Equilibrium of prestressed networks. IMA J. Appl. Math. 48(2), 195-215 (1992)

94. Neff, P., Münch, I.: Simple shear in nonlinear Cosserat elasticity: bifurcation and induced microstructure. Contin. Mech. Thermodyn. 21(3), 195-221 (2009)

95. dell'Isola, F., Seppecher, P., Madeo, A.: How contact interactions may depend on the shape of Cauchy cuts in Nth gradient continua: approach “à la D’Alembert”. Z. Angew. Math. Phys. ZAMP 63(6), 1119-1141 (2012)

96. dell'Isola, F., Steigmann, D.: A two-dimensional gradient-elasticity theory for woven fabrics. J. Elast. OnlineFirst. doi: 10.1007/s10659-014-9478-1 
97. Boutin, C., Soubestre, J.: Generalized inner bending continua for linear fiber reinforced materials. Int. J. Solids Struct. 48(34), 517-534 (2011)

98. Soubestre, J., Boutin, C.: Non-local dynamic behavior of linear fiber reinforced materials. Mech. Mater. 55, 16-32 (2012)

99. Pideri, C., Seppecher, P.: A second gradient material resulting from the homogenization of an heterogeneous linear elastic medium. Contin. Mech. Thermodyn. 9(5), 241-257 (1997)

100. Camar-Eddine, M., Seppecher, P.: Determination of the closure of the set of elasticity functionals. Arch. Ration. Mech. Anal. 170(3), 211-245 (2003)

101. dell'Isola, F., Madeo, A., Placidi, L.: Linear plane wave propagation and normal transmission and reflection at discontinuity surfaces in second gradient 3D continua, Zeitschrift fur Angewandte Mathematik und Mechanik. ZAMM 92(1), 52$71(2011)$

102. Steigmann, D., Pipkin, A.: Finite deformations of wrinkled membranes. Q. J. Mech. Appl. Math. 42(3), 427-440 (1989)

103. Oliveto, G., Cuomo, M.: Incremental analysis of plane frames with geometric and material nonlinearities. Eng. Struct. 10(1), 2-12 (1988)

104. Cuomo, M., Ventura, G.: Complementary energy approach to contact problems based on consistent augmented Lagrangian formulation. Math. Comput. Model. 28(4-8), 185-204 (1998)

105. Michel, J. C., Moulinec, H., Suquet, P.: A computational method based on augmented Lagrangians and fast fourier transforms for composites with high contrast. Comput. Model. Eng. Sci. CMES 1(2), 79-88 (2000)

106. Bilotta, A., Formica, G., Turco, E.: Performance of a high-continuity finite element in three-dimensional elasticity. Commun. Numer. Methods Eng. 26(9), 1155-1175 (2010)

107. Greco, L., Cuomo, M.: B-spline interpolation of Kirchhoff-love space rods. Comput. Methods Appl. Mech. Eng. 256, 251269 (2013)

108. Cazzani, A., Malagù, M., Turco, E.: Isogeometric analysis of plane curved beams. Math. Mech. Solids. OnlineFirst. doi:10. 1177/1081286514531265 (20 Apr 2014)

109. Greco, L., Cuomo, M.: An implicit g1 multi patch b-spline interpolation for Kirchhoff-love space rod. Comput. Methods Appl. Mech. Eng. 269, 173-197 (2014)

110. Forest, S.: Mechanics of generalized continua: construction by homogenization. Le Journal de Physique IV 08(PR4), 3948 (1998)

111. Neff, P., Forest, S.: A geometrically exact micromorphic model for elastic metallic foams accounting for affine microstructure. Modelling, existence of minimizers, identification of moduli and computational results. J. Elast. 87(2-3), 239$276(2007)$

112. Turco, E.: Identification of axial forces on statically indeterminate pin-jointed trusses by a nondestructive mechanical test. Open Civil Eng. J. 7(1), 50-57 (2013)

113. Moncada, M., Cognini, F., De Angelis, U., Ferrara, D., De Santis, G., Pilloni, L., Barbieri, G., Rinaldi, A.: Experimental assessment of damage mechanisms in both one-piece and welded aluminum foam sandwich beams. Int. Conf. Damage Mech.-ICDM, Belgrade, Serbia (2012)

114. Barbieri, G., Rinaldi, A., Cognini, F., Moncada, M., De Angelis, U., Ferrara, D., De Santis, G., Pilloni, L., Tatì, A.: The mechanical behavior of aluminum foam-based composite beams made as one-piece or by welding joining of two pieces. In: VI Aluminium Surface Science \& Technology Symposium-(VI ASST), Sorrento, Italy (2012)

115. Federico, S., Gasser, T.: Nonlinear elasticity of biological tissues with statistical fibre orientation. J. R. Soc. Interface 7(47), 955-966 (2010)

116. Lekszycki, T., dell'Isola, F.: A mixture model with evolving mass densities for describing synthesis and resorption phenomena in bones reconstructed with bio-resorbable materials. Zeitschrift Fur Angewandte Mathematik Und Mechanik ZAMM 92(6), 426-444 (2012)

117. Madeo, A., Lekszycki, T., dell'Isola, F.: A continuum model for the bio-mechanical interactions between living tissue and bio-resorbable graft after bone reconstructive surgery. Comptes Rendus - Mécanique 339(10), 625-640 (2011)

118. Andreaus, U., Giorgio, I., Lekszycki, T.: A 2D continuum model of a mixture of bone tissue and bio-resorbable material for simulating mass density redistribution under load slowly variable in time. Zeitschrift fur Angewandte Mathematik und Mechanik ZAMM. OnlineFirst. doi:10.1002/zamm.201200182 (26 Aug 2014)

119. Andreaus, U., Giorgio, I., Madeo, A.: Modeling of the interaction between bone tissue and resorbable biomaterial as linear elastic materials with voids. Z. Angew. Math. Phys. ZAMP. OnlineFirst. doi:10.1007/s00033-014-0403-z (23 Feb 2014)

120. Vakakis, A., Gendelman, O., Bergman, L., McFarland, D., Kerschen, G., Lee, Y.: Nonlinear Targeted Energy Transfer in Mechanical and Structural Systems. Springer-Verlag, New York (2008)

121. Pham, T., Pernot, S., Lamarque, C.: Competitive energy transfer between a two degree-of-freedom dynamic system and an absorber with essential nonlinearity. Nonlinear Dyn. 62(3), 573-592 (2010)

122. Sestieri, A., Carcaterra, A.: Space average and wave interference in vibration conductivity. J. Sound Vib. 263(3), 475491 (2003)

123. Roveri, N., Carcaterra, A., Akay, A.: Energy equipartition and frequency distribution in complex attachments. J. Acoust. Soc. Am. 126(1), 122-128 (2009)

124. Roveri, N., Carcaterra, A., Akay, A.: Vibration absorption using non-dissipative complex attachments with impacts and parametric stiffness. J. Acoust. Soc. Am. 126(5), 2306-2314 (2009)

125. Porfiri, M., dell'Isola, F., Santini, E.: Modeling and design of passive electric networks interconnecting piezoelectric transducers for distributed vibration control. Int. J. Appl. Electromagn. Mech. 21(2), 69-87 (2005)

126. Alessandroni, S., dell'Isola, F., Porfiri, M.: A revival of electric analogs for vibrating mechanical systems aimed to their efficient control by pzt actuators. Int. J. Solids Struct. 39(20), 5295-5324 (2002)

127. Andreaus, U., dell'Isola, F., Porfiri, M.: Piezoelectric passive distributed controllers for beam flexural vibrations. J. Vib. Control 10(5), 625-659 (2004)

128. dell'Isola, F., Maurini, C., Porfiri, M.: Passive damping of beam vibrations through distributed electric networks and piezoelectric transducers: prototype design and experimental validation. Smart Mater. Struct. 13(2), 299-308 (2004) 
129. D’Annibale, F., Rosi, G., Luongo, A.: Linear stability of piezoelectric-controlled discrete mechanical systems under nonconservative positional forces. Meccanica, OnlineFirst. doi:10.1007/s11012-014-0037-4 (29 Aug 2014)

130. D'Annibale, F., Rosi, G., Luongo, A.: On the failure of the 'similar piezoelectric control' in preventing the loss of stability caused by nonconservative positional forces. Z. Angew. Math. Phys. ZAMP. OnlineFirst. doi:10.1007/s00033-014-04777 (23 Oct 2014)

131. Rinaldi, A., Correa-Duarte, M., Salgueirino-Maceira, V., Licoccia, S., Traversa, E., Davila-Ibanez, A., Peralta, P., Sieradzki, K.: In-situ micro-compression tests of single core-shell nanoparticles. Acta Mater. 58(19), 6474-6486 (2010)

132. Rinaldi, A.: Effects of dislocation density and sample-size on plastic yielding at the nanoscale: a Weibull-like framework. Nanoscale 3(11), 4817-4823 (2011)

133. Rinaldi, A., Peralta, P., Sieradzki, K., Traversa, E., Licoccia, S.: Dislocation density on sample-size dependent plasticity in nanoscale pillars and buttons. J. Nanomech. Micromech. 2(3), 42-48 (2012)

134. Araneo, R., Rinaldi, A., Notargiacomo, A., Bini, F., Marinozzi, F. Lovat, G., Celozzi, S.: Effect of the scaling of the mechanical properties on the performances of $\mathrm{ZnO}$ piezo-semiconductive nanowires. AIP Conference Proceedings, vol.1603, pp.14-22 (2014)

135. Araneo, R., Bini, F., Pea, M., Notargiacomo, A., Rinaldi, A., Lovat, G., Celozzi, S.: Current-voltage characteristics of ZnO nanowires under uniaxial loading. IEEE Trans. Nanotechnol. 13(4), 724-735 (2014)

136. Abeyaratne, R., Knowles, J.: Evolution of Phase Transitions. A Continuum Theory. Cambridge University Press, Cambridge (2006). ISBN:9780521380515

137. Berezovski, A., Engelbrecht, J., Maugin, G.: Numerical Simulation of Waves and Fronts in Inhomogeneous Solids. World Scientific, Singapore (2008) ISBN:978-981-283-267-2

138. Bhattacharya, K.: Phase boundary propagation in a heterogeneous body. Proc. R. Soc. Lond. A 455, 757-766 (1999)

139. Bhattacharya, K., James, R.: The material is the machine. Science 307(5706), 53-54 (2005)

140. Li, Z., Sun, Q.: The initiation and growth of macroscopic martensite band in nano-grained NiTi microtube under tension. Int. J. Plast. 18(11), 1481-1498 (2002)

141. Miyazaki, S., Fu, Y., Huang, W. (eds.): Thin Film Shape Memory Alloys: Fundamentals and Device Applications. Cambridge University Press, Cambridge (2009) ISBN:9780521885768

142. Eremeyev, V., Pietraszkiewicz, W.: The non-linear theory of elastic shells with phase transitions. J. Elast. 74(1), 6786 (2004)

143. Pietraszkiewicz, W., Eremeyev, V., Konopinska, V.: Extended non-linear relations of elastic shells undergoing phase transitions. Zeitschrift Fur Angewandte Mathematik Und Mechanik ZAMM 87(2), 150-159 (2007)

144. Eremeyev, V., Pietraszkiewicz, W.: Phase transitions in thermoelastic and thermoviscoelastic shells. Arch. Mech. 61(1), 4167 (2009)

145. Eremeyev, V., Pietraszkiewicz, W.: Thermomechanics of shells undergoing phase transition. J. Mech. Phys. Solids 59(7), 1395-1412 (2011)

146. Contro, R., Poggi, C., Cazzani, A.: Numerical analysis of fire effects on beam structures. Eng. Comput. 5(1), 53-58 (1988)

147. Cazzani, A., Rovati, M.: Sensitivity analysis and optimum design of elastic-plastic structural systems. Meccanica 26(23), 173-178 (1991)

148. Cazzani, A., Contro, R., Corradi, L.: On the evaluation of the shakedown boundary for temperature-dependent elastic properties. Eur. J. Mech. A/Solids 11, 539-550 (1992)

149. Lakes, R., Rosakis, P., Ruina, A.: Microbuckling instability in elastomeric cellular solids. J. Mater. Sci. 28(17), 4667$4672(1993)$

150. F. A. S. L. Eremeev, V.A.: Nonuniqueness and stability in problems of equilibrium of elastic two-phase bodies. Dokl. Phys. 48(7), 359-363 (2003)

151. Steigmann, D.: Frame-invariant polyconvex strain-energy functions for some anisotropic solids. Math. Mech. Solids 8(5), 497-506 (2003)

152. Yeremeyev, V., Freidin, A., Sharipova, L.: The stability of the equilibrium of two-phase elastic solids. J. Appl. Math. Mech. 71(1), 61-84 (2007)

153. Pampolini, G., Del Piero, G.: Strain localization in open-cell polyurethane foams: experiments and theoretical model. J. Mech. Mater. Struct. 3(5), 969-981 (2008)

154. Truskinovsky, L., Vainchtein, A.: Kinetics of martensitic phase transitions: lattice model. SIAM J. Appl. Math. 66(2), 533553 (2005)

155. Truskinovsky, L., Vainchtein, A.: Quasicontinuum models of dynamic phase transitions. Contin. Mech. Thermodyn. 18, 121 (2006)

156. Ferretti, M., Piccardo, G.: Dynamic modeling of taut strings carrying a traveling mass. Contin. Mech. Thermodyn. 25, 469488 (2013) 